\title{
Preparing Primary Care for COVID-20
}

\author{
Mohammed K. Ali, MD, MSc, MBA ${ }^{1,2}$, Deep J. Shah, MD, MSc ${ }^{3}$, and Carlos del Rio, MD ${ }^{2,4}$

'Department of Family and Preventive Medicine, School of Medicine, Emory University Atlanta, GA, USA; ' ${ }^{H}$ Hubert Department of Global Health, Rollins School of Public Health, Emory University Atlanta, GA, USA; ${ }^{3}$ Division of General Internal Medicine and Geriatrics, Department of Medicine, School of Medicine, Emory University Atlanta, GA, USA; ${ }^{4}$ Division of Infectious Diseases, Department of Medicine, School of Medicine, Emory UniversityAtlanta, GA, USA.

J Gen Intern Med

DOI: $10.1007 / \mathrm{s} 11606-020-05945-5$

(c) Society of General Internal Medicine 2020

$\mathrm{T}$ he COVID-19 crisis prompted government and healthcare leaders to marshal resources from every corner of the nation to cope with surge demand. The focus has been on increasing floor and intensive care bed capacity, ventilator caches, and stores of lifesaving personal protective equipment (PPE). Similarly, public health measures such as broad testing and social distancing are being implemented with vigor. Beyond these on-the-ground battles, however, there is a larger war that policymakers and health system leaders need to consider: SARS-CoV-2 and respiratory pathogens are not one-time occurrences. The pandemic, along with its health and economic devastation, may recur in the second half of 2020 , or possibly sooner as Southern hemisphere countries face winter from May onwards. Populations remain vulnerable as we are unlikely to see vaccines, reliable therapies, and universal testing anytime soon. Early, well-planned, and well-resourced pandemic preparedness for the next wave(s) is much needed and we need a transformation in America for how we prepare. We argue that our health and economy may be best served by strengthening the role of primary care in tackling the next wave(s), thereby reducing the burden on public health, emergency, and inpatient services. We discuss what will be required to equip primary care practices to lead early management and disease notification in the short time frame before the next wave.

Primary care, when paired with robust public health infrastructure, is the linchpin of any modern healthcare system's ability to track and manage disease pandemics, ${ }^{1}$ but has largely been sidelined in the current pandemic. Primary care physicians (PCPs) in the USA are uniquely positioned to shoulder greater responsibility during the next phase of COVID-19. PCPs are trusted resources for and claim strong, longitudinal relationships with patients. PCPs can leverage multiple established avenues to reach patients as well as close contacts especially given the evolving focus on isolation and contact

Received May 5, 2020

Accepted May 22, 2020 tracing. An additional channel of communication has been added with the rapid implementation of telemedicine and remote care monitoring during the current crisis. Moreover, primary care clinics - including single and networked primary care offices, federally qualified health centers, and frequently visited retail clinics - are well distributed across the country. Finally, primary care offices are capable of managing patients flow across home, clinic, hospital, and post-acute care; recent lifting of data sharing restrictions by the U.S. Department of Health and Human Services will enhance this coordination.

There is no equivalent comparison with the COVID-19 pandemic, given its lethal combination of transmissibility, unpredictable course, and case fatality. However, there are valuable lessons from previous outbreaks (e.g., H1N1) and the role that PCPs play in seasonal influenza management. The majority of influenza-like illnesses (ILI) are assessed and managed in primary care. Observational data have shown that early testing and treatment for seasonal flu and H1N1 are associated with less pneumonias, repeat visits, hospitalizations, and mortality. ${ }^{2,3}$ As testing and perhaps a therapy with some benefit become more broadly available, these can be administered in primary care. And even if not, PCPs can administer more supportive care in their offices as PPE becomes more available. Whenever SARS-CoV-2 vaccines ultimately enter clinical practice, PCPs can assume an even greater role through prevention. Robust primary care infrastructure for ILI facilitates access and surge capacity; enables timely diagnosis and treatment; and could reduce health disparities in the incidence of pneumonias, hospitalizations, and related morbidity and mortality. ${ }^{4,5}$

The clock is already ticking. The tight timeline is further complicated by limited reliable data. It is imperative, though, that policymakers begin to outline the basic requirements if primary care is to play a greater role in saving lives and reducing the burden on healthcare systems. Specifically, the primary care community needs the following (Table 1):

(1) definitions and standards for evaluating and managing cases;

(2) outbreak protocols giving primary care offices a central role in early detection, local safety, and surveillance;

(3) clinical decision-making tools including rapid tests and prediction rules; and

(4) supportive policies. 


\section{Table 1 Components of Primary Care Pandemic Preparedness}

\begin{tabular}{l}
\hline \hline Definitions \\
"At-risk" patients \\
Protocols \\
Telephone screening \\
Telemedicine visits \\
Infection control in outpatient settings and healthcare worker safety \\
Treatments and quarantines for people under investigation and mild \\
cases \\
Clinical decision-making tools \\
Rapid testing and associated sensitivity and specificity \\
Clinical scoring tools (based on symptoms, vital signs, \\
basic laboratory data) \\
Thresholds for imaging (x-rays, computed tomography) \\
Thresholds for urgent referral \\
Supportive policies \\
Surveillance and reporting system activation \\
Availability of PPE \\
Financial support \\
Staff training \\
Workforce augmentation \\
\hline
\end{tabular}

To obtain and operationalize definitions, protocols, and clinical tools, we propose efficient accumulation of data with an emphasis on the role of asymptomatic carriers and prognostic factors for early symptomatic cases - those most likely to present to the outpatient setting. Leadership and support from federal agencies can promote generous health system data sharing and rapid translation of findings to workable standards. Quickly leveraging electronic medical records and online search data will expedite identification of risk factors for infection and transmission; time course of illness; and risk factors for hospitalization, intensive care admission, mortality, and/or healthy discharge. For efficient use of data as it is released, we propose a task force bringing together a coalition of professional societies representing PCPs in internal and family medicine, pediatrics, gynecology, and infectious disease and public health experts. This group should review the data by mid-summer and develop iterative consensus care algorithms at both state and federal levels by early fall.

Lastly, policymakers will need to facilitate pathways to manage the next wave. When deployed properly, PCPs can assume leadership roles in disease surveillance in partnership with local health departments and hospitals. ${ }^{6}$ Local reporting systems should be able to handle high volume; "stress testing" the systems through drills between now and then will be invaluable. At the national level, higher primary care participation in the CDC's Outpatient Influenza-like Illness Surveillance Network (ILINet) will help identify regional hotspots where focused testing, contact tracing, and isolation in the next COVID outbreak can support rapid containment. ${ }^{7}$ Widespread testing and syndromic surveillance could prevent the economy from shutting down again.

Federal and state guidance for PCPs to invest in preparedness measures alone will be insufficient. Opt-out instead of opt-in policies will lead to wider adoption. However, at a minimum, PCPs will need guarantees of support in the form of PPE and funding indexed on panel size. These could be executed through memoranda of understanding. Payments will need to focus on business continuity through payroll and fixed cost support - similar to the Small Business Administration's Paycheck Protection Program loans. In addition, participating primary care clinics may benefit from training for primary office care personnel and/or workforce support to meet surge demand. Travel nurses, typically utilized in hospitals, may prove valuable coaches for medical assistants less familiar with strict infection control protocols.

To be sure, this cannot be callous deployment of front-line physicians and much relies on the availability of PPE and effective anti-viral therapies. That said, investing in primary care pandemic preparedness may enhance and reshape relationships between primary care and government, payer, medical supplier, and hospital stakeholders. These changes may have benefits beyond pandemic response(s), heralding a renaissance in the role of primary care in America's health landscape. PCPs serve as quarterbacks in coordinating their patients' care and have a unique vantage point and patient trust, which has been underused in the COVID-19 detection, prevention, and management responses. This pandemic might be the stimulus for rethinking healthcare delivery and leaning into the notion that primary care is the foundation of a strong healthcare system.

Acknowledgments: The authors would like to graciously acknowledge and thank Robert M. McLean, MD, from the American College of Physicians, Philadelphia, PA, for the critical review of the manuscript.

Corresponding Author: Mohammed K. Ali, MD, MSc, MBA; Hubert Department of Global Health, Rollins School of Public Health, Emory University Atlanta, GA, USA (e-mail: mkali@emory.edu).

\section{Compliance with Ethical Standards:}

Conflict of Interest: The authors declare that they do not have a conflict of interest.

\section{REFERENCES}

1. A World At Risk: Annual report on global preparedness for health emergencies. 2019. (Accessed April 6, 2020, at https://apps.who.int/ gpmb/assets/annual_report/GPMB_annualreport_2019.pdf.)

2. Yu H, Liao Q, Yuan Y, et al. Effectiveness of oseltamivir on disease progression and viral RNA shedding in patients with mild pandemic 2009 influenza A H1N1: opportunistic retrospective study of medical charts in China. Bmj 2010;341:c4779.

3. Wang C-B, Chiu M-L, Lin P-C, et al. Prompt Oseltamivir Therapy Reduces Medical Care and Mortality for Patients With Influenza Infection: An Asian Population Cohort Study. Medicine (Baltimore) 2015;94:e1070.

4. Inpatient Hospital Stays and Emergency Department Visits Involving Influenza, 2006-2016. HCUP Statistical Brief \#253. October 2019. (Accessed April 4, 2020, at www.hcup-us.ahrq.gov/reports/statbriefs/ sb253-Influenza-Hospitalizations-ED-Visits-2006-2016.pdf.)

5. Hadler JL, Yousey-Hindes K, Perez A, et al. Influenza-Related Hospitalizations and Poverty Levels - United States, 2010-2012. MMWR Morb Mortal Wkly Rep 2016;65:101-5.

6. van den Wijngaard CC, van Asten L, Meijer A, et al. Detection of excess influenza severity: associating respiratory hospitalization and mortality data with reports of influenza-like illness by primary care physicians. American journal of public health 2010;100:2248-54.

7. A National COVID-19 Surveillance System: Achieving Containment. 2020. (Accessed April 18, 2020, at https://healthpolicy.duke.edu/sites/default/ files/atoms/files/covid-19_surveillance_roadmap_final.pdf.)

Publisher's Note: Springer Nature remains neutral with regard to jurisdictional claims in published maps and institutional affiliations. 